\title{
Investigação de sísmica de reflexão rasa em depósitos Quaternários e Terciários que apresentam indícios de ação neotectônica na cidade de Taubaté/SP Shallow seismic reflection survey in Quaternary and Tertiary deposits with evidence of neotectonics action in Taubaté city, Sao Paulo state, Brazil
}

\author{
Victor Coelho Pereira*1, Renato Luiz Prado', Liliana Alcazar Diogo', Oleg Bokhonok², Nayara de Oliveira Stenico ${ }^{3}$ e Allan
}

Segovia ${ }^{4},{ }^{1}$ AG-USP ${ }^{2}$ |peci-Unisantos ${ }^{3}$ FEC-Unicamp ${ }^{4}$ Alura

Copyright 2021, SBGf - Sociedade Brasileira de Geofísica

This paper was prepared for presentation during the $17^{\text {th }}$ International Congress of the Brazilian Geophysical Society held in Rio de Janeiro, Brazil, 16-19 August 2021.

Contents of this paper were reviewed by the Technical Committee of the $17^{\text {th }}$ International Congress of the Brazilian Geophysical Society and do not necessarily represent any position of the SBGf, its officers or members. Electronic reproduction or storage of any part of this paper for commercial purposes without the written consent storage of any part of this paper for commercial
of the Brazilian Geophysical Society is prohibited.

\begin{abstract}
Although the seismic reflection method is commonly employed for imaging geological structures to subsides studies of the geological evolution of sedimentary basins, usually their use is limited to the deep scale of the oil/gas investigations. This paper presents an analysis of seismic reflection data (synthetic and experimental) conducted with the objective of mapping a shallow structure, or some evidence of it, in Quaternary and Tertiary sediments of the Taubate Basin, São Paulo, Brazil, originated by neotectonics activity. From the synthetic data is discussed some limitations regarding the vertical resolution and the minimum depth of the geological subsurface that can be recovered, considering the seismic wave frequency content. The experimental data shows that the seismic investigation can bring valuable subsidies for comprehension of the neotectonics evolution of the study area.
\end{abstract}

\section{Introdução}

A principal motivação desta pesquisa é a de gerar subsídios para o estudo da tectônica do Quaternário na parte central da Bacia de Taubaté. Os métodos tradicionais de estudo da neotectônica incluem usualmente a análise dos depósitos sedimentares, estruturas presentes, morfologia dos terrenos e imagens de sensoriamento remoto. A inclusão de outros métodos investigativos, como os sísmicos, pode auxiliar enormemente, especialmente pelo fato de poder gerar perfis contínuos da subsuperfície.

O método de sísmica de reflexão já é empregado usualmente no mapeamento de estruturas mais profundas (maior que $100 \mathrm{~m}$ de profundidade, podemos dizer).

No que diz respeito à Bacia Sedimentar de Taubaté, já há levantamentos sísmicos na sua porção Central e Nordeste, realizados pela Petrobras em 1988, interpretados por diferentes pesquisadores (Marques, 1990; Fernandes, 1993; Cogné et al., 2013). Porém, todos trazem informações da estratigrafia e estruturas de profundidades superiores a $100 \mathrm{~m}$.
Em se tratando das estruturas geradas pela tectônica do Quaternário na Bacia de Taubaté, o desafio para o emprego dos métodos geofísicos está relacionado principalmente ao alcance da resolução (lateral e em profundidade), uma vez que os rejeitos das falhas podem ser da ordem de poucos metros e presentes a pouca profundidade como diversas pesquisas já relataram (Riccomini, 1989; Mancini, 1995).

Com a mesma motivação aqui apresentada, Lucena (2016) empregou o método MASW (Multichanel Analysis of Surface Waves) na investigação de estrutura rúptil observada em uma das margens do Rio Una (município de Taubaté, SP) que parece afetar o colúvio quaternário e os sedimentos fluviais da Formação Pindamonhangaba, terciários.

Este trabalho apresenta resultados preliminares de dados sintéticos (reflexão) e dados coletados na área (aquisição CMP - Common Mid Point) visando subsidiar o estudo dessa possível estrutura e discutir as potencialidades do emprego do método na investigação rasa de estruturas geológicas mais recentes na Bacia de Taubaté.

\section{Tectônica do Quaternário na Bacia de Taubaté}

A interpretação de estruturas tectônicas rúpteis observadas na bacia mostram a ocorrência de pelo menos três regimes tectônicos que atuaram no Quaternário (Mancini \& Riccomini, 1994; Mancini, 1995). O mais antigo, de caráter transcorrente dextral com compressão NW-SE tem seu início registrado no Neógeno e finalizado no Quaternário. No Holoceno, ocorre uma mudança no regime tectônico que passa a ser extensional, com direção geral E-W, e finalmente compressivo, com direção E-W.

O regime extensional no Holoceno foi o responsável por várias falhas normais observadas na Formação Pindamonhangaba (microfalhas até grandes estruturas com rejeitos de alguns metros de extensão). Essas falhas colocaram em contato tectônico os sedimentos da Formação Pindamonhangaba e os dos depósitos do Quaternário (Mancini, 1995) e poderiam explicar a ocorrência de uma estrutura em hemi-graben na área de estudo, discutida por Lucena (2016).

\section{Geologia da área de estudos e dados sintéticos}

Os levantamentos sísmicos dessa pesquisa ocorreram em área localizada no município de Taubaté. Nesse local afloram sedimentos da Formação Resende (Grupo 
Taubaté), Formação Pindamonhangaba, e depósitos colúvio-aluviais do Quaternário (Fig. 1).

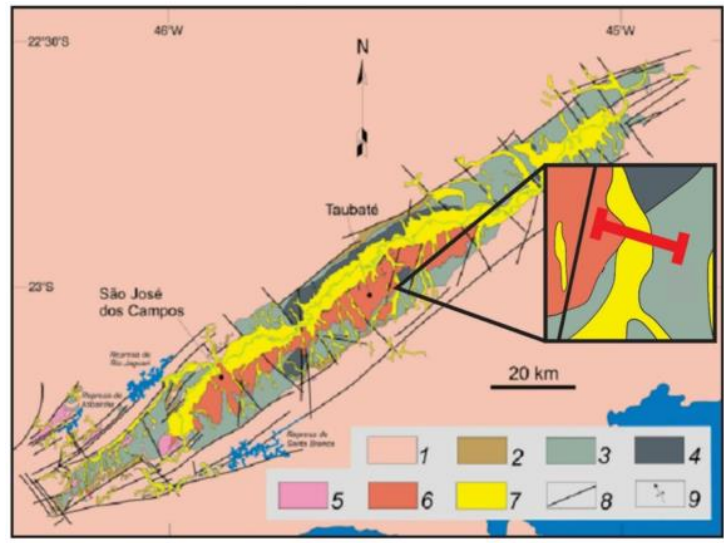

Figura 1 - Mapa geológico da Bacia de Taubaté com a localização do perfil esquemático da Figura 2 (elaborado a partir do modelo apresentado por Lucena (2016)). 1) Embasamento; 2) Formação Resende (sistema de leques aluviais proximais); 3) Formação Resende (sistema de leques aluviais medianos a distais associados a planície aluvial de rios entrelaçados); 4) Formação Tremembé; 5) Formação São Paulo; 6) Formação Pindamonhangaba; 7) Sedimentos quaternários; 8) Falhas cenozoicas; 9) Eixo de dobras principais (Modificado de Riccomini (1989) e Riccomini et al. (2004) apud Lucena (2016)).

O modelo geológico/estrutural da área de estudos utilizado para a geração dos sismogramas sintéticos partiu do modelo elaborado por Lucena (2016). É de um hemi-graben com camadas mergulhando para NW. O modelo de Lucena (2016) foi concebido com base nos estudos da geologia e evolução litoestratigráfica da bacia (Riccomini, 1889; Riccomini et al., 2004), na observação de estruturas que indicam movimentação de falha na margem direita do Rio Una, na geomorfologia do local e nas informações geofísicas resultantes do levantamento MASW que realizou. Aqui foram acrescentadas informações extraídas dos resultados dos levantamentos geofísicos de Hamada (2018), método TDEM (Time Domain Electromagnetic), e Rangel (2018), métodos geoelétricos, além de informações do perfil do poço 3500051142, localizado próximo ao local do ensaio (SIAGAS - CPRM).

O modelo apresentado na Figura 2 considera a espessura dos sedimentos do Quaternário entre 10 e 30 metros, sobrepostos sobre sedimentos da Formação Pindamonhangaba (20 metros de espessura), cuja base se assenta sobre os sedimentos do Grupo Taubaté. Dentro do pacote de sedimentos do Grupo Taubaté, considerou-se a existência de uma camada de arenitos (25 metros de espessura) entre argilitos, informação extraída de perfil do poço 3500051142 (SIAGAS CPRM). O embasamento é representado pelo semiespaço a 300 metros de profundidade.

Os estudos de modelagem foram realizados posteriormente à aquisição dos dados. $O$ modelo geológico 2D, que considera camadas homogêneas e isotrópicas, foi criado pelo programa Trimodel e os sismogramas sintéticos (aquisição CMP) pelo programa Triseis, ambos do pacote Seismic Unix - SU (Forel et al., 2005).

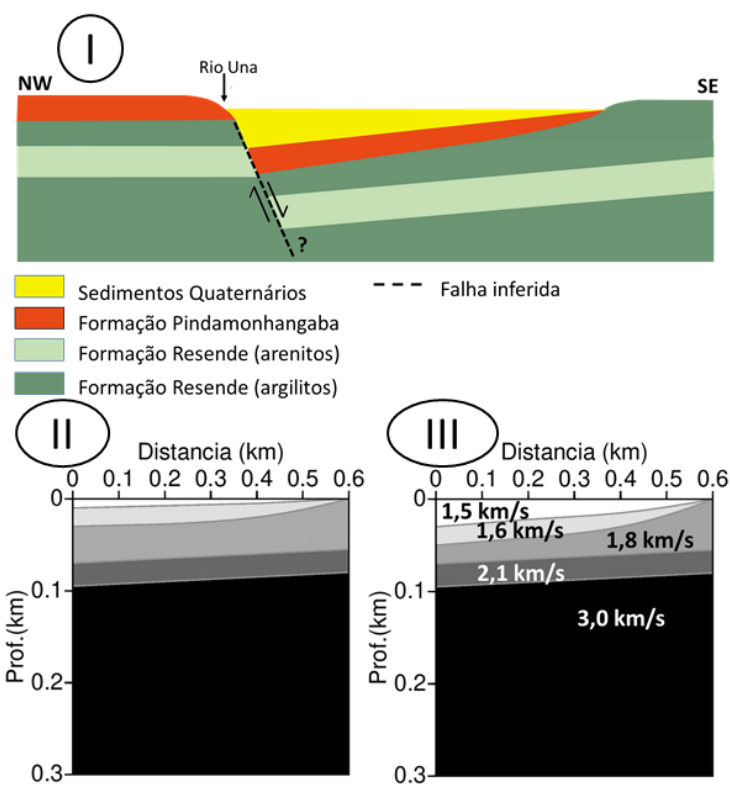

Figura 2 - I) Modelo geológico/estrutural para a área de estudos. II e III) Modelos adotados para a geração dos sismogramas sintéticos com as velocidades de propagação de ondas adotadas.

O objetivo dos testes de modelagem foi o de verificar a resolução vertical do método para mapear os estratos presentes considerando diferentes frequências dominantes do sinal sísmico, 30 e $100 \mathrm{~Hz}$, e as espessuras aqui consideradas para os diferentes estratos, assim como comparar os tempos de percurso obtidos na modelagem com os observados na seção sísmica empilhada. As pequenas espessuras dos estratos, assim como a grandeza dos rejeitos de falhas esperados para os eventos do Quaternário são limitadores importantes para o emprego do método de sísmica de reflexão. Nos dois ensaios sísmicos cujos resultados são apresentados neste trabalho foram adotados parâmetros de aquisição distintos (que serão melhor detalhados a frente). No ensaio A foi empregado um compactador de solos como fonte geradora de ondas e geofones de $14 \mathrm{~Hz}$ de frequência, e no ensaio $B$ foi empregada uma marreta como fonte geradora de ondas e geofones de $40 \mathrm{~Hz}$. Daí a razão de gerarmos os sismogramas sintéticos com wavelets de frequências dominantes de 30 e $100 \mathrm{~Hz}$.

Os sismogramas sintéticos foram gerados considerando um arranjo de 96 geofones, espaçados de 1 metro e offset mínimo de 1 metro, com fonte e arranjo de geofones se deslocando de 2 metros a cada tiro. 
A Figura 3 apresenta os sismogramas sintéticos de dois conjuntos de tiros considerando espessuras de 10 e 30 metros para os sedimentos do Quaternário e frequências dominantes de 30 e $100 \mathrm{~Hz}$. As Figuras 4 e 5 apresentam as respectivas seções empilhadas sintéticas para as mesmas condições de espessuras e frequências dominantes.

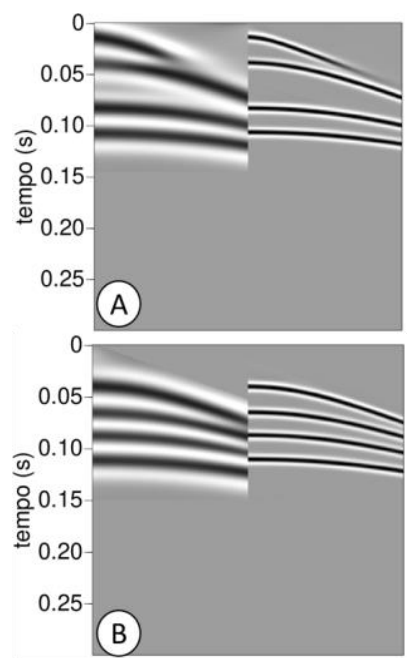

Figura 3 - Sismogramas sintéticos de conjuntos de tiros considerando espessura de 10 metros $(A)$ e 30 metros (B) para os sedimentos do Quaternário e frequências dominantes de 30 e $100 \mathrm{~Hz}$.

Da análise das imagens das duas figuras pode-se concluir, considerando os pequenos rejeitos esperados para as falhas e consequentemente as pequenas espessuras dos sedimentos do Quaternário, que a frequência dominante do sinal é fator fundamental para o imageamento desse estrato, ou seja, deve-se empregar fontes que preferencialmente gerem ondas de espectro de frequência elevado, assim como geofones de frequência natural de $40 \mathrm{~Hz}$ ou superior. Nos sismogramas gerados com wavelets de frequência de 30 $\mathrm{Hz}$ já não é possível se distinguir o contato do Quaternário com a Formação Pindamonhangaba quando esta se encontra a profundidade de $10 \mathrm{~m}$. Da mesma forma, os refletores relacionados ao topo e base da Formação Pindamonhangaba ocorrem em tempos menores que 0,09 segundo, o que também é fator limitante considerando as sempre presentes interferências do groundroll nessa janela de tempo nos dados de sísmica de reflexão rasa.

\section{Dados experimentais: parâmetros de aquisição e resultados}

Nesta seção são apresentados os resultados dos ensaios realizados em duas seções, A e B, que possuem diferentes azimutes (Fig. 6) e foram adquiridas empregando-se diferentes parâmetros de aquisição.

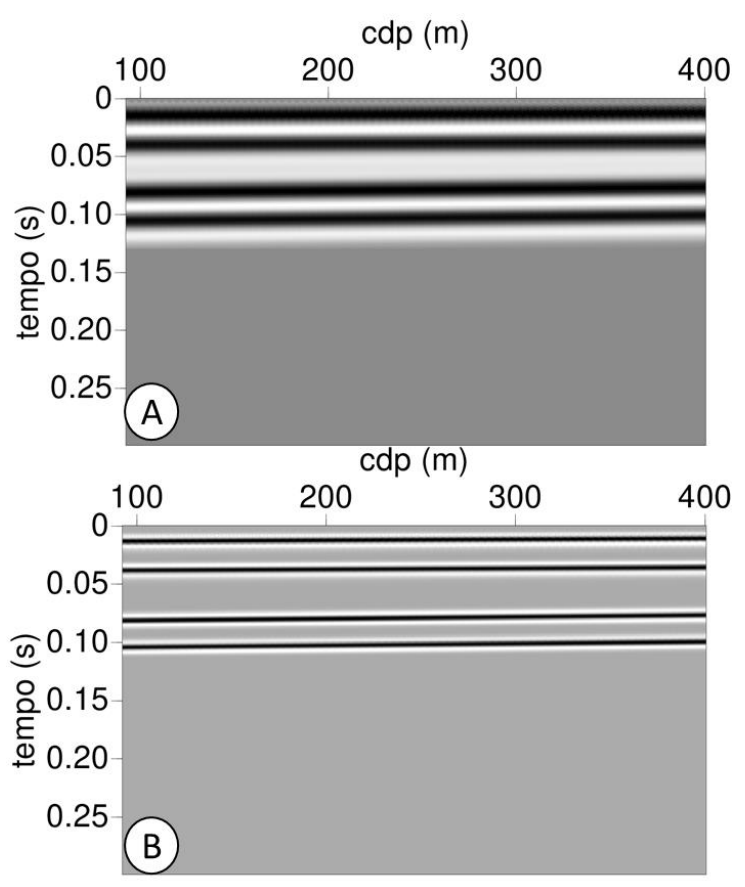

Figura 4 - Seções empilhadas obtidas a partir dos dados sintéticos considerando espessuras de $10 \mathrm{~m}$ para a camada do Quaternário e frequências dominantes de A) $30 \mathrm{~Hz}$ e B) $100 \mathrm{~Hz}$.

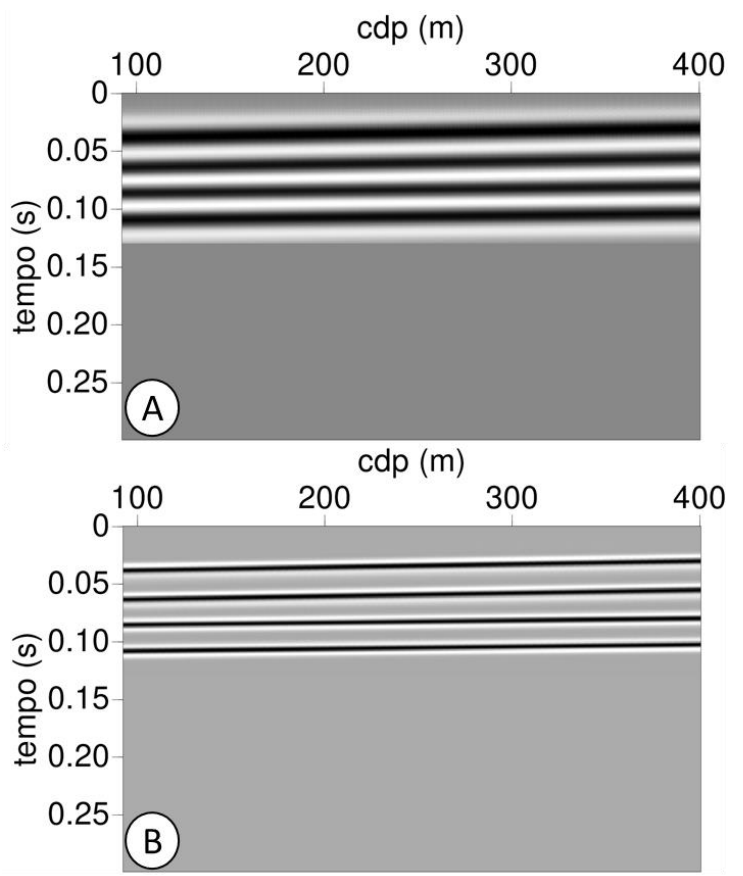

Figura 5 - Seções empilhadas obtidas a partir dos dados sintéticos considerando espessuras de $30 \mathrm{~m}$ para a camada do Quaternário e frequências dominantes de $A$ ) $30 \mathrm{~Hz}$ e B) $100 \mathrm{~Hz}$ 


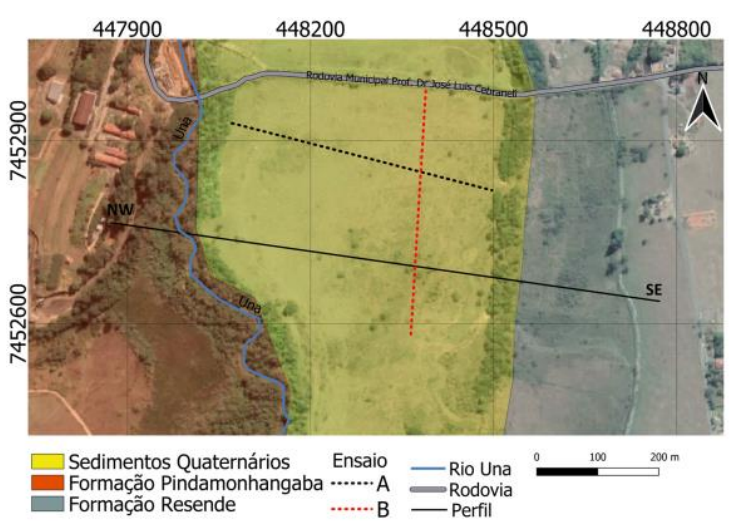

Figura 6. Esboço de mapa geológico simplificado sobreposto à imagem de satélite (coordenadas UTM) do local dos ensaios (Disponível em: http://earth.google.com. Acessado em 10/02/21).

$\mathrm{Na}$ aquisição dos dados da seção $\mathrm{A}$ foram empregados um compactador de solo como fonte sísmica, através da técnica Mini-Sosie (Barbier et al., 2006), 96 geofones de $14 \mathrm{~Hz}$ de frequência espaçados de 2 metros. A aquisição CMP dos dados de reflexão foi executada mantendo-se os geofones fixos e a fonte deslocando-se de 2 metros a cada ponto de tiro. Após cada sequência de 48 tiros, 48 geofones eram movidos para a parte final do arranjo, e assim sucessivamente. Os offsets mínimos variaram de 96 a 2 metros a cada sequência de tiros.

$\mathrm{Na}$ aquisição dos dados da seção $\mathrm{B}$, empregou-se uma marreta como fonte sísmica, 96 geofones de $40 \mathrm{~Hz}$ espaçados de 1 metro. A aquisição CMP foi executada mantendo-se os geofones fixos e a fonte deslocando-se de 2 metros a cada ponto de tiro. Após cada sequência de 12 tiros, 24 geofones eram movidos para a parte final do arranjo. Os offsets mínimos variaram de 97 a 75 metros a cada sequência de tiros. Em ambos os ensaios foi utilizado o sismógrafo Geode (Geometrics Inc.) de 96 canais.

As seções empilhadas em tempo com as interpretações preliminares são apresentadas nas Figuras 7 e 8 . São interpretados os refletores que apresentam maior continuidade nas seções.

As limitações impostas pelo groundroll nos dados experimentais (conjuntos de tiro) permitiram somente 0 mapeamento de refletores com tempos duplos de reflexão iguais ou superiores a $0,1 \mathrm{~s}$, aqui interpretados como pertencentes ao Grupo Taubaté.

Embora não tenha sido realizada correção estática de campo nos conjuntos de tiro, pois o terreno onde foram realizados os ensaios tem topografia plana e a camada de solos apresenta pequena espessura até o nível d'água, consideramos que eventuais efeitos de estática são desprezíveis para a análise das seções empilhadas.

Assim, pode-se observar na seção A que os refletores na sua porção NW apresentam mergulho para NW (em detalhe na Figura 9).
A não continuidade lateral de alguns refletores pode estar associada a variações laterais das características dos sedimentos ou eventualmente à existência de pequenos deslocamentos dos estratos na geração do hemi-graben.

Os refletores observados na seção B (Fig. 8) estão menos perturbados, mais contínuos. A maior continuidade pode ser devido ao fato desta seção estar posicionada ortogonalmente ao sentido do mergulho das camadas na estrutura hemi-graben considerado no modelo estrutural.

\section{Comentários finais}

Os resultados obtidos pelos dados sintéticos indicam que a caracterização dos pequenos rejeitos esperados para as falhas e consequentemente pequenas espessuras dos sedimentos do Quaternário dependem da frequência dominante da onda sísmica registrada, portanto das características da fonte e da resposta dos geofones. Quanto maior for a frequência, mais viável é a caracterização das estruturas em subsuperfície esperadas para essa área de estudos. Nos sismogramas sintéticos produzidos com wavelets de frequência de 30 $\mathrm{Hz}$ não é possível distinguir o contato entre os sedimentos Quaternários e a Formação Pindamonhangaba quando se assume que este contato está a $10 \mathrm{~m}$ de profundidade.

Para os dados experimentais, mesmo não tendo sido possível mapear a base dos sedimentos do Quaternário e da Formação Pindamonhangaba, pode-se observar pequenos mergulhos dos refletores da porção superior do Grupo Taubaté. Considerando que as falhas que resultaram na estrutura em hemi-graben (e consequente rejeito) se estendem até às porções superiores do Grupo Taubaté, o diagnóstico dos efeitos da neotectônica podem ser inferidos a partir dos mergulhos observados nesses estratos sísmicos.

Para as porções mais rasas (digamos, inferiores a 100 $\mathrm{m})$, levantamentos de sísmica de refração parecem ser os mais indicados.

A continuidade desses estudos prevê o processamento e interpretação dos dados de uma terceira seção de sísmica de reflexão, já adquirida, e dos dados de sísmica de refração da seção A aqui apresentada.

Espera-se que a integração de todos esses dados venha trazer mais esclarecimentos sobre a estrutura estudada. 


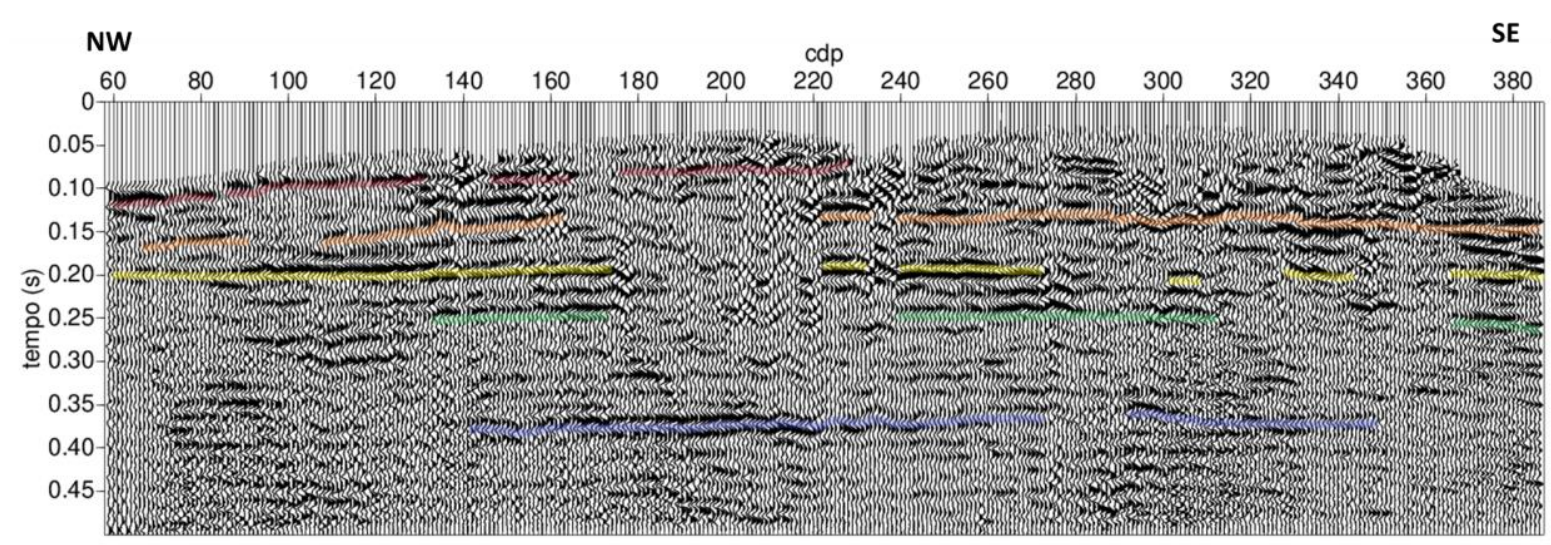

Figura 7- Seção empilhadas em tempo para o ensaio A e os refletores interpretados.

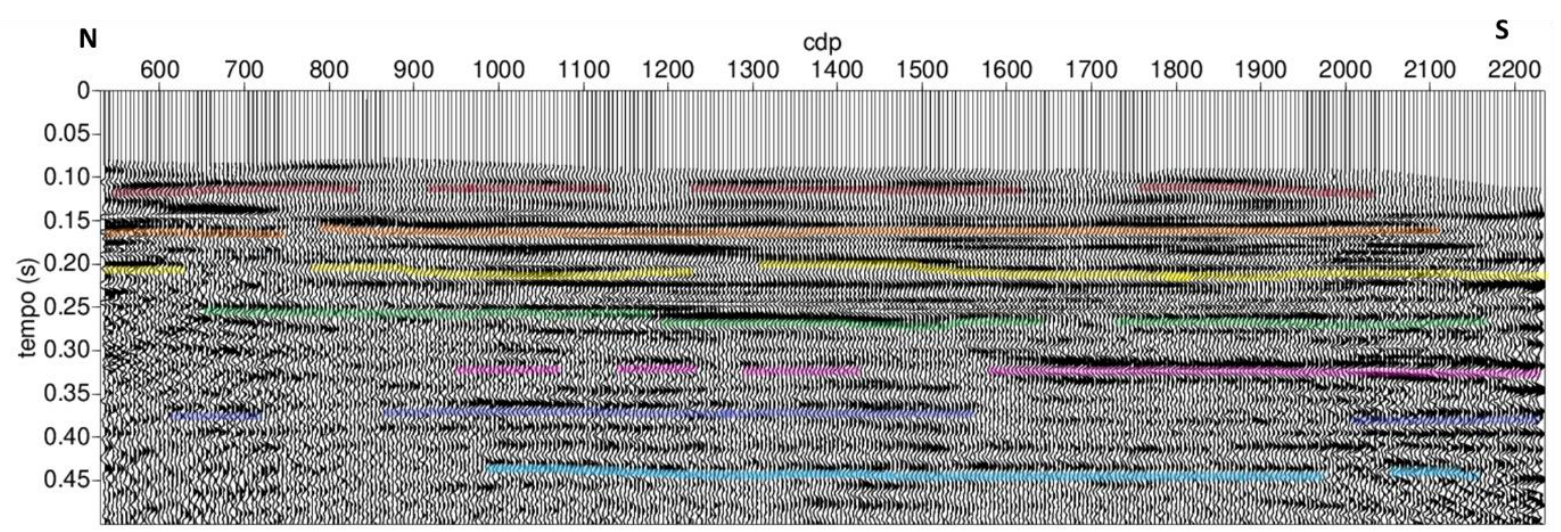

Figura 8 - Seção empilhadas em tempo para o ensaio B e os refletores interpretados.

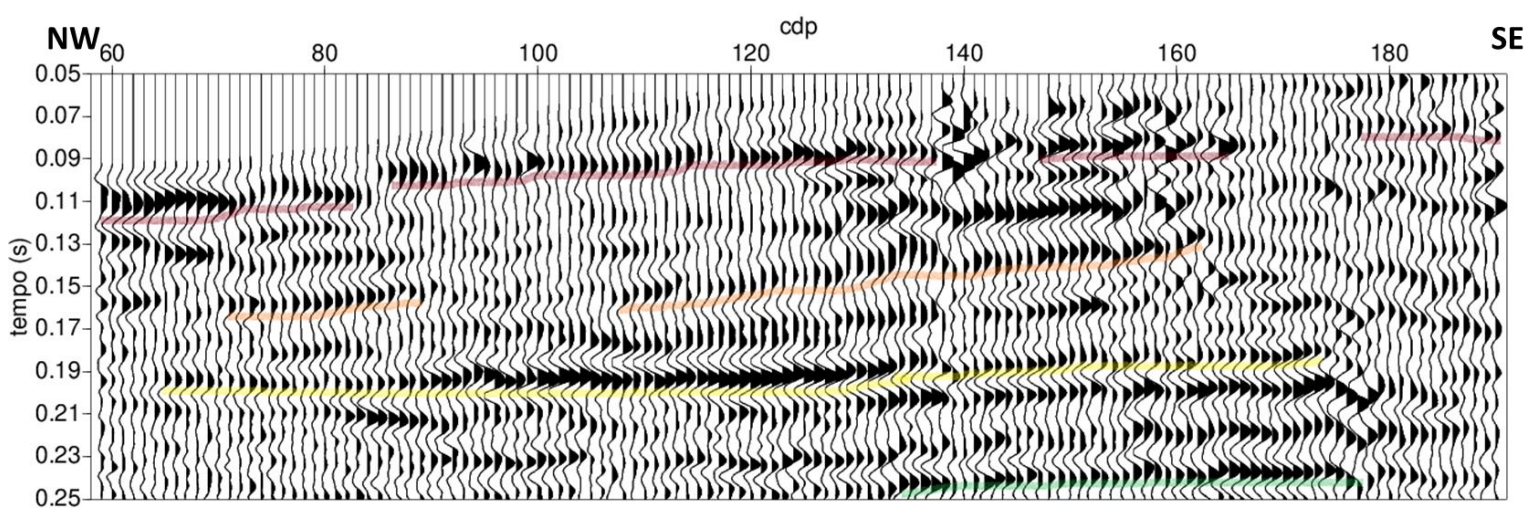

Figura 9 - Em detalhe as inclinações dos refletores da seção empilhada A. 


\section{Agradecimentos}

VCP agradece a CAPES pela concessão da bolsa de estudos. Os autores agradecem ao CNPq pelo apoio à realização dos ensaios de campo (Projeto CNPq PVE 406653/2013-5), ao IGc-USP pelo apoio à fase inicial dos levantamentos de campo, e ao IAG-USP pelo apoio com toda infraestrutura: pessoal, equipamentos, veículos e laboratórios.

\section{Referências}

BARBIER, M.G., BONDON, P., MELLINGER, R., VIALLIX, J.R. 2006. Mini-Sosie for land seismology. Geophysical Prospecting 24(3), 518-526.

COGNÉ, N., COBBOLD, P. R., RICCOMINI, C. \& GALLAGHER, K. 2013. Basin (Southeastern Brazil): Insights from regional seismic profiles and outcrop data. Journal of South American Earth Sciences, 42, 194-204.

FERNANDES, F. L. 1993. Arcabouço estrutural e evolução da Bacia de Taubaté-SP. Dissertação de Mestrado. Departamento de Geologia, Universidade Federal de Ouro Preto, Ouro Preto, 225p.

FOREL, D; BENZ, T \& PENNINGTON, Wayne D. 2005. Seismic Data Processing with Seismic Un* $x$ : A 2D Seismic Data Processing Primer. Society of Exploration Geophysicists.

HAMADA, L. R., PORSANI, J. L., BORTOLOZO, C. A., \& RANGEL, R. C. 2018. TDEM and VES soundings applied to a hydrogeological study in the central region of the Taubaté Basin, Brazil. First Break, 36(8), 49-54.

LUCENA, R. F. 2016. Avaliações e testes dos métodos MASW e ReMi por meio do tratamento de dados empíricos e sintéticos em um programa de processamento e inversão desenvolvido em MATLAB e sua implicação em um problema geológico na Bacia de Taubaté. Dissertação de Mestrado. Instituto de Astronomia, Geofísica e Ciências Atmosféricas, Universidade de São Paulo, São Paulo, 212p.

MANCINI, F. \& RICCOIMINI, C. 1994. Estilos estruturais da Formação Pindamonhangaba, Bacia de Taubaté, SP. In: Congresso Brasileiro de Geologia, 38. Balneário de Camboriú, SC.

MANCINI, F. 1995. Estratigrafia e Aspectos da Tectônica Deformadora da Formação Pindamonhangaba, Bacia de Taubaté, SP. Dissertação de Mestrado. Instituto de Geociências, Universidade de São Paulo, São Paulo, $107 p$.

MARQUES, A. 1990. Evolução tectono-sedimentar e perspectivas exploratórias da Bacia de Taubaté, São Paulo, Brasil. Boletim de Geociências da Petrobrás, 4(3), 253-262.

RANGEL, R. C., PORSANI, J. L., BORTOLOZO, C. A., \& HAMADA, L. R. 2018. Electrical Resistivity Tomography and TDEM Applied to Hydrogeological Study in Taubaté Basin, Brazil. International Journal of Geosciences, 9(2), 119-130.

RICCOMINI, C. 1989. O rift continental do sudeste do Brasil. Tese de Doutorado. Instituto de Geociências, Universidade de São Paulo, São Paulo, 256p.

RICCOMINI, C., SANT'ANNA, L. G. \& FERRARI, A. L. 2004. Evolução geológica do rift continental do sudeste do Brasil. Geologia do continente Sul-Americano: evolução da obra de Fernando Flávio Marques de Almeida. 383-405.

SIAGAS - CPRM. CPRM: Brazilian Geological Service; SIAGAS - Groundwater Information System. Available online:

http://siagasweb.cprm.gov.br/layout/pesquisa_complexa.p hp. Accessed: 19 February, 2021 